\title{
Holographic grating stability: influence of 4,4'-azobis (4-cyanopentanoic acid) on various spatial frequencies
}

\author{
E. Fernández,,$^{1,3, \star}$ R. Fuentes,,$^{1,3}$ M. Ortuño, ${ }^{2,3}$ A. Beléndez, ${ }^{2,3}$ and I. Pascual ${ }^{1,3}$ \\ 'Departamento de Óptica, Farmacología y Anatomía, Universidad de Alicante, Apartado 99, E-03080 Alicante, Spain \\ ${ }^{2}$ Departamento de Física, Ingeniería de Sistemas y Teoría de la Señal, Universidad de Alicante, \\ Apartado 99, E-03080 Alicante, Spain \\ ${ }^{3}$ Instituto Universitario de Física Aplicada a las Ciencias y las Tecnologías, Universidad de Alicante, \\ Apartado 99, E-03080 Alicante, Spain \\ ${ }^{*}$ Corresponding author: elena.fernandez@ua.es
}

Received 9 May 2013; revised 9 July 2013; accepted 3 August 2013;

posted 5 August 2013 (Doc. ID 190217); published 29 August 2013

\begin{abstract}
This paper presents the results obtained when holographic gratings were stored with a spatial frequency of 954 and 2663 lines/mm in transmission geometry and 4600 lines/mm in reflection geometry in a polyvinyl alcohol/acrylamide-based material. Photopolymers are materials that give good results at low frequencies, but their diffraction efficiency (DE) decreases at high frequencies. A chain transfer agent, 4,4'-azobis (4-cyanopentanoic acid) (ACPA) was incorporated in the material composition to improve spatial resolution. Furthermore, a curing process was applied to the stored gratings in order to maintain the DE stable over time. The DE and shrinkage for symmetric holographic transmission and reflection gratings were measured to evaluate their quality and quantify the improvement produced by ACPA. (C) 2013 Optical Society of America

OCIS codes: (090.0090) Holography; (090.7330) Volume gratings; (090.2900) Optical storage materials; (160.5470) Polymers.

http://dx.doi.org/10.1364/AO.52.006322
\end{abstract}

\section{Introduction}

In the last decade, holography has acquired great importance since holographic devices can store information throughout the volume of the material [1-4], thereby increasing the storage capacity in comparison with two-dimensional devices that only store information on the surface. Pioneering companies in this field, such as Bayer MaterialScience and InPhase [3-5], even came together to create the Tapestry $\mathrm{TM}$, the first prototype holographic optical storage system that is being used by leading

$1559-128 \mathrm{X} / 13 / 256322-10 \$ 15.00 / 0$

(C) 2013 Optical Society of America companies and is capable of storing from $200 \mathrm{~GB}$ to $1.6 \mathrm{~TB}$ in a disk $130 \mathrm{~mm}$ in diameter.

A fundamental element of these devices is the recording material where the information is registered. Research groups around the world are concentrating all their efforts on obtaining a suitable material for this purpose [6-10]. It is important to obtain a material with a high refractive index modulation and high sensitivity as well as good optical quality. In addition, more recently, efforts have been made to find materials with high resolution $[11,12]$. This type of hologram has also been used for other applications, such as a visual indicator of environmental humidity $[\underline{13}, 14]$.

Photopolymers are materials capable of storing gratings with a diffraction efficiency (DE) close to 
$100 \%$ for a spatial frequency of 1000 lines $/ \mathrm{mm}$. However, for holographic data storage, a material that is capable of resolving higher spatial frequencies is needed so that a greater amount of data may be stored. When working with higher spatial frequencies, photopolymers are no longer able to resolve the frequencies correctly, thus leading to a decrease in DE. For this reason, in this work the standard composition of a polyvinyl alcohol (PVA)-acrylamide based photopolymer is modified by introducing a chain transfer agent, namely $4,4^{\prime}$-azobis (4-cyanopentanoic acid) (ACPA) [15], that increases the resolution of the photopolymer and therefore increases the DE of the stored gratings. In particular, diffraction gratings of 954,2663 , and 4600 lines $/ \mathrm{mm}$ are stored in two types of polyvinyl alcohol/ acrylamide (PVA/AA)-based photopolymers-one with ACPA and the other without. In this way, the influence of ACPA can be analyzed by comparing the DEs of these stored gratings.

To store the above spatial frequencies in the material holographically, two different geometries are used to record the gratings. Gratings with 954 and 2663 lines $/ \mathrm{mm}$ are stored in transmission geometry and gratings with 4600 lines/mm are stored in reflection geometry.

The most widely studied branch of holography, which has given rise to a large number of papers, is transmission holography $[16,17]$. However, leading companies such as Bayer are beginning to conduct studies aimed at using reflection holography [18]. In order to obtain more compact systems it would be interesting to design reflection holographic memories to which the current technology for reading CDs and DVDs designed for reflection holograms may be applied. Furthermore, reflection geometry has the advantage that higher spatial frequencies may be obtained than in transmission geometry; thus a larger amount of data may be stored. The disadvantage of storing higher spatial frequencies is that materials with high $\mathrm{DE}$ in transmission geometry have lower $\mathrm{DE}$ in reflection geometry, hence the requirement to explore new materials and/or modify existing ones, altering their composition in order to resolve higher spatial frequencies. The DE and shrinkage for symmetric holographic transmission and reflection gratings were measured to evaluate their quality and quantify the improvement produced by ACPA.

Moreover, it is important that the information stored in the material endures over time. To achieve this, after storage, gratings are subjected to a curing process that eliminates the surplus dye and prevents further reactions taking place in the material. The $\mathrm{DE}$ is thus prevented from decreasing with time. Gallego et al. [19] compared three ways to remove residual monomer and dye, thus stabilizing the stored gratings: using incoherent white light, coherent green light, and heating the grating. Jenney fixed the gratings using ultraviolet light [20]. In this paper we use an Aktiprint-M Compact $\overline{\mathrm{UV}}$ Curing Unit Bridge emitting ultraviolet light to fix the gratings.
The curing process is carried out in the gratings stored in the two photopolymer compositions mentioned above. It will be seen that ACPA has a positive effect on maintaining the DE stable since gratings stored in the photopolymer, with ACPA loss a smaller percentage of $\mathrm{DE}$ than do those in the photopolymer without ACPA.

\section{Experimental Setup}

\section{A. Preparation of the Material}

The holograms are recorded in a PVA/AA based photopolymer. The standard composition of this material contains $\mathrm{AA}$ as the polymerizable monomer, triethanolamine (TEA) as radical generator, PVA as binder, and yellowish eosin (YE) as sensitizer. Table 1 shows the concentrations of the components of this material (composition 1). Considering the versatility of photopolymers when new components are added to their composition, a chain transfer agent (the ACPA) was decided to add to the standard composition of the used photopolymer. This component produces a new reaction in the formation of the grating that can be explained as follows: A growing macroradical reacts with the chain transfer agent, producing a deactivation of the growing macroradical, so a new radical can start a new polymer chain. The main effect of this polymerization reaction is the reduction of the polymer chain length, which in turn can improve the material response to the recorded spatial frequency. Therefore, the ACPA, which acts as chain transfer agent, was added to the standard composition to improve the spatial resolution of the material. The concentrations of the components are shown in Table 1 (composition 2).

To obtain these two materials with the compositions in Table 1 , first a solution of PVA in water was prepared by heating and once all the PVA had dissolved it was allowed to cool. Simultaneously, a solution of AA and TEA was prepared in water. These two components were mixed in the proportions necessary to obtain the concentrations of composition 1 (Table 1). Finally, YE was added to the solution under red light because the material is not sensitive at this wavelength. In the case of composition 2 (Table 1 ), ACPA was also added to the solution and stirred until completely dissolved. The mixture was then deposited by gravity on a $22 \mathrm{~cm} \times 40 \mathrm{~cm}$ glass plate and left in the dark for 1 day approximately to allow the water to evaporate in conditions of temperature, $T=20^{\circ} \mathrm{C}$, and relative humidity, $\mathrm{RH}=40 \%$, in order

Table 1. Concentrations of the Photopolymer Compositions

\begin{tabular}{lcc}
\hline & Composition 1 & Composition 2 \\
\hline PVA & $8.26 \% \mathrm{~m} / \mathrm{v}$ & $8.26 \% \mathrm{~m} / \mathrm{v}$ \\
AA & $0.44 \mathrm{M}$ & $0.44 \mathrm{M}$ \\
TEA & $0.20 \mathrm{M}$ & $0.20 \mathrm{M}$ \\
YE & $2.4 \times 10^{-4} \mathrm{M}$ & $2.4 \times 10^{-4} \mathrm{M}$ \\
ACPA) & - & $0.015 \mathrm{M}$ \\
\hline
\end{tabular}


to obtain $70 \pm 5 \mu \mathrm{m}$ thick plates. Once dry, the glass was cut into squares of $6 \mathrm{~cm} \times 6 \mathrm{~cm}$.

After manufacturing the material, its optical properties, including refractive index and absorption spectrum of each composition, were measured.

The refractive index of the material was calculated using an Abbe's refractometer. For composition 1, a refractive index of $n_{532}=1.515 \pm 0.001$ was obtained for a wavelength of $532 \mathrm{~nm}$ and $n_{633}=1.510 \pm 0.001$ for a wavelength of $633 \mathrm{~nm}$. And for composition 2, a $n_{532}=1.518 \pm 0.001$ and a $n_{633}=1.512 \pm 0.001$ were obtained. These two refractive indexes will be used to calculate the spatial period of the recorded gratings and the Bragg wavelength in Section 2.B.

Figure 1 shows the absorption spectrum of the material versus the wavelength for the two compositions. As can be seen, the plot exhibits maximum absorption at the recording wavelength of $\lambda=532 \mathrm{~nm}$. Consequently, the material composition is optimized so that the material is as sensitive as possible at the recording wavelength. In the range between $\lambda=570 \mathrm{~nm}$ and $\lambda=650 \mathrm{~nm}$, the transmittance is minimum and thus the material does not absorb or absorbs very little.

The geometries for the grating recording and reconstruction were chosen so that the Bragg angle (in transmission geometry) or Bragg wavelength (in reflection geometry) would appear in this nonabsorbing spectral range (see Section 2.B).

\section{B. Holographic Setup}

In this section, the holographic setup used to store holographic transmission gratings with a spatial frequency of 954 to 2663 lines/mm is described together with that used to store gratings of about 4600 lines/mm.

First, Fig. 2 shows the experimental setup used to store holographic transmission gratings. In the recording stage, two beams-the object beam and the reference beam-interfere at an angle of $\theta$ with the normal to form the hologram. As the two beams can be considered plane waves, a diffraction grating is obtained. The laser used in the recording stage was a Nd: $\mathrm{YVO}_{4}$ laser (Coherent Verdi V2), emitting a beam with a wavelength of $532 \mathrm{~nm}$, at which the material is sensitive (Fig. 1). To store the diffraction

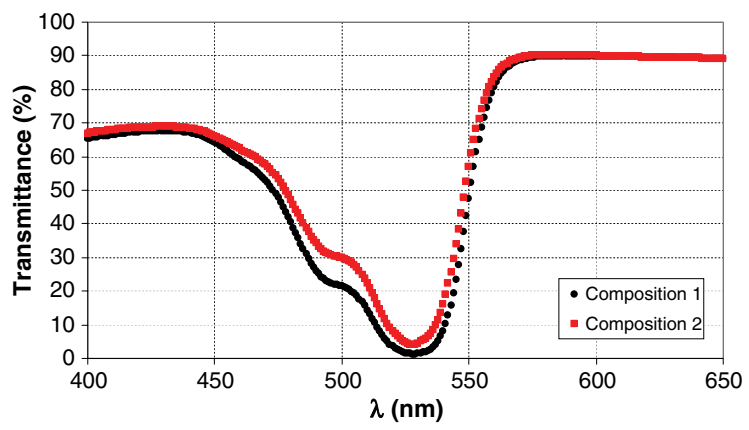

Fig. 1. Transmission spectrum of the unexposed photopolymer plate: composition 1 with black circles and composition 2 with red squares.

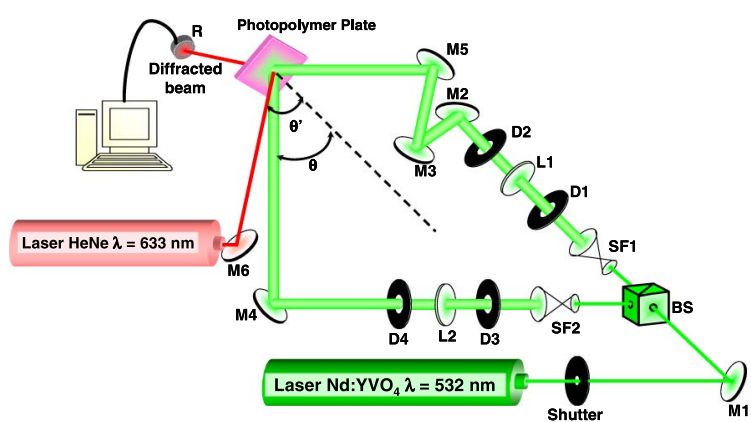

Fig. 2. Experimental setup for transmission gratings. Mi, mirrors; BS, beam splitter; $\mathrm{Li}$, lenses; $\mathrm{SFi}$, microscope objective lens and pinhole; Di, diaphragms; R, radiometer.

grating, the vertically polarized laser beam was split into two beams with a beam-splitter, passed through a microscope objective and a pinhole to expand and filter the beams, and through a series of lenses and diaphragms to collimate them with the desired diameter $(1 \mathrm{~cm})$. Finally, some mirrors directed the beams to produce the interference at the desired angle on the recording material. The total intensity of the recording beams was $5 \mathrm{~mW} / \mathrm{cm}^{2}$.

The recording angle was modified in the experimental setup according to Eq. (1) to achieve the two spatial frequencies mentioned in Section 1

$$
\sin \theta=\frac{\lambda}{2 \Lambda},
$$

where $1 / \Lambda$ is the spatial frequency and $\lambda$ is the wavelength measured in air [21].

In the reconstruction stage, a $\mathrm{He}-\mathrm{Ne}$ laser with a wavelength of $633 \mathrm{~nm}$ was used to reconstruct the holographic grating since the material is not sensitive at this wavelength (Fig. 1). The laser beam falls on the material at an angle of $\theta^{\prime}$ calculated from Eq. (1). The power of the diffracted beam was measured with a radiometer and the $\mathrm{DE}$ of the grating is defined as the ratio of the power of the diffracted beam to the power of the incident beam.

A recording angle of $14.7^{\circ}$ was used to achieve a spatial frequency of 954 lines $/ \mathrm{mm}$ and an angle of $17.6^{\circ}$, calculated from Eq. (1), was used to reconstruct the grating. In the case of a spatial frequency of 2663 lines $/ \mathrm{mm}$, the recording angle was $45.1^{\circ}$ and the grating was reconstructed with an angle of $57.4^{\circ}$.

Second, Fig. 3 shows the experimental setup used to store holographic reflection gratings with a spatial frequency of 4600 lines $/ \mathrm{mm}$. Holographic reflection gratings were stored using a $\mathrm{Nd}: \mathrm{YVO}_{4}$ laser (Coherent Verdi V2) with a wavelength of $532 \mathrm{~nm}$, at which the material was sensitive (see Fig. 1). A symmetric geometry was used in order to obtain gratings with interference fringes parallel to the recording material. The setup is shown in Fig. 3 . Again, the vertical polarized beam emitted by the laser was split into two beams with a beam-splitter. Each beam was expanded and filtered using a microscope 
objective and a pinhole and a series of lenses and diaphragms collimated beams with the desired diameter. The total intensity of the recording beams was $24 \mathrm{~mW} / \mathrm{cm}^{2}$ with an intensity ratio of $1: 1$. The two laser beams were spatially overlapped at the recording medium intersecting at an angle of $63.3^{\circ}$ (measured in air).

Using the refractive index of the material with composition $1\left(n_{532}=1.515\right)$, the intersection angle in air, $\theta$, and the recording wavelength, $\lambda=532 \mathrm{~nm}$, the theoretical spatial period of the grating, $\Lambda_{\mathrm{th}}$, was $0.2174 \mu \mathrm{m}$, applying Eq. (2) (therefore the spatial frequency of the recorded reflection gratings was 4600 lines $/ \mathrm{mm}$ ). And using the refractive index of the material with composition $2 \quad\left(n_{532}=1.518\right)$, and the same intersection angle and recording wavelength, the theoretical spatial period was $\Lambda_{\text {th }}=0.2168 \mu \mathrm{m}$ and its spatial frequency was 4613 lines/mm:

$$
\Lambda=\frac{\lambda}{2 \sqrt{n^{2}-\sin ^{2} \theta}}
$$

In the reconstruction stage, the $\mathrm{DE}$ was measured by a double-beam spectrophotometer, which measures the transmittance of the photopolymer versus the wavelength.

As discussed in Section 3, the graphs obtained with the transmission geometry are different from those obtained with the reflection geometry due to the way of obtaining them. For the transmission geometry, the $\mathrm{DE}$ is obtained as a function of the reconstruction angle by using a $\mathrm{He}-\mathrm{Ne}$ laser with a wavelength of $633 \mathrm{~nm}$, as shown in Fig. 2. For the reflection geometry, a double beam spectrophotometer was used to reconstruct the stored gratings, since the diffracted beam overlaps the reflected beam at the interface air-material (because of the symmetric grating geometry), so in this case the grating cannot be reconstructed as in the transmission geometry.

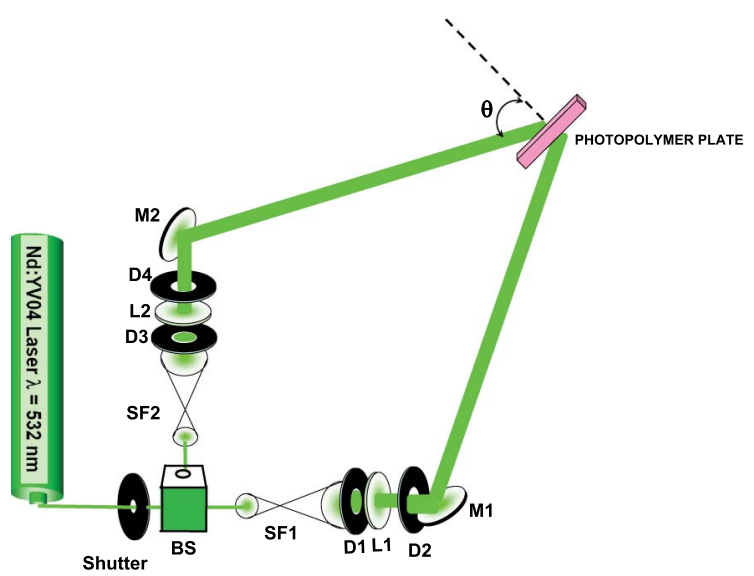

Fig. 3. Experimental setup for reflection gratings. BS, beam splitter; $\mathrm{Mi}$, mirror; Li, lens; Di, diaphragm; $\mathrm{SFi}$, microscope objective lens and pinhole.

\section{Shrinkage Measurement}

During the photopolymerization process, the conversion of monomer molecules into a polymer network is accompanied by close packing of the growing chains and a subsequent reduction in volume. This phenomenon is known as photopolymerization shrinkage.

However, this reduction in volume can also be produced by other processes. In hydrophilic materials like the one presented in this work, the evaporation of the water contained in its composition can also produce this reduction in volume, producing also a shrinkage process. Whether the shrinkage is produced by a polymerization process, as if it is produced by another process, the final result is a shift of the Bragg planes or a change in the spatial period of the grating, and the way to calculate the shrinkage for both cases is detailed below.

For transmission gratings, shrinkage of the material can produce a shift in the reconstruction angle, which may be due to a shift in the grating fringes or a change in the spatial period. In the general case of a grating with slanted planes, Gallo and Verber [22] demonstrated that the shift angle, $\Delta \phi$, of the slanted fringes of the grating produced after shrinkage is

$$
\phi^{\prime}=\phi+\Delta \phi=\tan ^{-1}\left[\frac{\tan \phi}{\left(1-S_{\mathrm{opt}}\right)}\right]
$$

where $\phi$ and $\phi^{\prime}$ are the slant angles of the fringes of the grating before and after shrinkage, respectively, and $S_{\mathrm{opt}}$ is the shrinkage coefficient.

For the particular case of a symmetrical transmission grating in which the fringes are not slanted, which is the case presented in this paper, the angle $\phi=\pi / 2$. Substituting this value in Eq. (3) gives $\phi^{\prime}=0$, that is, the shrinkage does not produce a shift in the grating fringes.

However, shrinkage may produce a change in the spatial period of the grating. Specifically, shrinkage occurs when the effective period of the grating recorded in the sample, $\Lambda_{\exp }$, is less than that defined by the geometrical conditions of the recording, $\Lambda_{\mathrm{th}}$. Shrinkage of the material induces a change in the fringe spacing $\Delta \Lambda=\Lambda_{\text {th }}-\Lambda_{\text {exp }}$. Criante et al. [23] define the optical shrinkage as the ratio between the spatial displacement of the period that is observed and the theoretical spatial period of the grating if there was not shrinkage, i.e.,

$$
S_{\mathrm{opt}}=\frac{\Lambda_{\mathrm{th}}-\Lambda_{\mathrm{exp}}}{\Lambda_{\mathrm{th}}}
$$

Solving the spatial period in Eq. (1) and substituting into Eq. (4), taking into account that $\theta_{\text {th }}=\theta^{\prime}$, that is the reconstruction angle, and $\theta_{\exp }=\theta^{\prime}+\Delta \theta$, where $\Delta \theta$ is the rotation of reconstruction angle produced by shrinkage, then Eq. (4) may be written as 


$$
S_{\mathrm{opt}}=\frac{\sin \left(\theta^{\prime}+\Delta \theta\right)-\sin \left(\theta^{\prime}\right)}{\sin \left(\theta^{\prime}+\Delta \theta\right)} .
$$

Considering the approximation that $\Delta \theta$ is small compared with $\theta^{\prime}$, Eq. (5) can be simplified to obtain Eq. (6). This equation will be used to calculate the optical shrinkage for transmission gratings:

$$
S_{\mathrm{opt}}=\frac{\Delta \theta}{\tan \left(\theta^{\prime}\right)} .
$$

In reflection gratings, shrinkage induces a change in the fringe spacing and the ultimate effect of the shrinkage is a shift in the wavelength at which the grating peak appears. To calculate the optical shrinkage, the spatial period of a symmetric reflection grating [Eq. (2)] is substituted in the definition of shrinkage [Eq. (4)]. The reconstruction angle is $\theta=0^{\circ}$ and the refractive index is the same as in the previous section. Therefore, after shrinkage, Eq. (ㅁ) becomes

$$
S_{\mathrm{opt}}=\frac{\lambda_{\mathrm{th}}-\lambda_{\mathrm{exp}}}{\lambda_{\mathrm{th}}}
$$

where $\lambda_{\text {th }}$ is the theoretical wavelength where the grating peak should appear if there was no shrinkage and $\lambda_{\exp }$ is the experimental wavelength where the grating peak actually appears. For composition 1 , substituting $n_{0}=1.515, \Lambda=\Lambda_{\text {th }}=0.2174 \mu \mathrm{m}$, and $\theta=\theta_{i}=0^{\circ}$ in Eq. (2), we obtain a theoretical wavelength $\lambda_{\mathrm{th}}=656 \mathrm{~nm}$. And for composition 2, substituting $n_{0}=1.518, \Lambda=\Lambda_{\text {th }}=0.2168 \mu \mathrm{m}$ and $\theta=\theta_{i}=0^{\circ}$ in Eq. (2) we obtain a theoretical wavelength $\lambda_{\text {th }}=655 \mathrm{~nm}$.

The optical shrinkage of the gratings was calculated for the three different spatial frequencies and is discussed in the results section.

\section{Results}

After adjusting the experiment setup, transmission gratings were recorded with two different spatial frequencies, 954 and 2663 lines $/ \mathrm{mm}$, and reflection gratings were recorded with a spatial frequency of 4600 lines $/ \mathrm{mm}$. The gratings were recorded in photopolymer plates with the two compositions in Table 1 . The only difference between these two compositions, as stated above, is that a chain transfer agent, ACPA, was added to composition 2 in an attempt to improve the resolution of the material.

It is important to record gratings with the maximum DE as possible. However, when information is stored, it should remain over time. For this reason, in this study a curing process was applied in order to maintain the DE stable. As mentioned in Section 2.A, one of the components of the photopolymer is a dye, YE, which absorbs photons, initiating the polymerization reaction. After recording the grating not all the dye in the photopolymer is consumed and the reaction continues if the hologram is exposed to incoherent light, for example by exposure to ambient light. In this situation the hologram progressively loses the $\mathrm{DE}$ and its response would not be reproducible in the time. One way to avoid this reaction is to remove the excess of dye after recording the grating, which was done in our experiment by means of a curing process.

Moreover, the curing process has another advantage: It minimizes the diffusion of polymer chains to the dark areas. Although such polymer spread is generally small, it becomes more intense in polymers with ACPA because they have smaller chains than when there is not ACPA, and therefore their diffusion is greater.

An Aktiprint-M Compact UV Curing Bridge Unit was used to carry out the curing process. This device emits ultraviolet radiation in the wavelength range between 300 and $400 \mathrm{~nm}$ with a power of $500 \mathrm{~W}$. It also has a conveyor belt where the recorded photopolymer is placed in order to receive the UV radiation during the time it takes to pass through the curing bridge. During this period of time the dye is removed, thereby stopping the polymerization reaction and preventing the loss of information. After recording the grating, each plate was exposed to the UV lamp. Experiments were made for different exposures and it was found that the optimal exposure was $1500 \mathrm{~J} / \mathrm{cm}^{2}$, with which all the dye was consumed with a minimum loss of $\mathrm{DE}$. Therefore, an exposure of $1500 \mathrm{~J} / \mathrm{cm}^{2}$ was used for curing.

As mentioned in Section 2.A, the material has the maximum absorption at $532 \mathrm{~nm}$ (Fig. 1). Therefore, to demonstrate that the excess of dye has been removed by the curing process, the nondestructive readout of the grating after curing is proved by using a green laser beam with a wavelength at which the material has strong absorption. The gratings were exposed with a green laser beam at $532 \mathrm{~nm} 6$ months after applying curing with an exposure of $100 \mathrm{~mJ} / \mathrm{cm}^{2}$, and finally they were reconstructed again.

Throughout the curing process, temperature and relative humidity were monitored to ensure that the possible changes in the $\mathrm{DE}$ of the stored grating were due to the curing process, and it was found that both the temperature and relative humidity remained constant. All the obtained results are shown in the next sections.

\section{A. Transmission Gratings with a Spatial Frequency of 954 lines/mm}

First, gratings were stored with a spatial frequency of 954 lines $/ \mathrm{mm}$ in photopolymers with compositions 1 and 2 in Table 1 . The results obtained are shown in Figs. $\underline{4}$ and $\underline{5}$. Each figure shows the DE of the stored grating just after recording (black circles), just after curing (red squares), $24 \mathrm{~h}$ after curing (blue triangles), 6 days after curing (green diamonds), and 6 months after curing and after an exposition of $100 \mathrm{~mJ} / \mathrm{cm}^{2}$ with a $532 \mathrm{~nm}$ laser beam (black asterisks). The zero of the abscissa axis corresponds to the Bragg angle for a wavelength of $633 \mathrm{~nm}$, which is $17.6^{\circ}$ for this spatial frequency. 


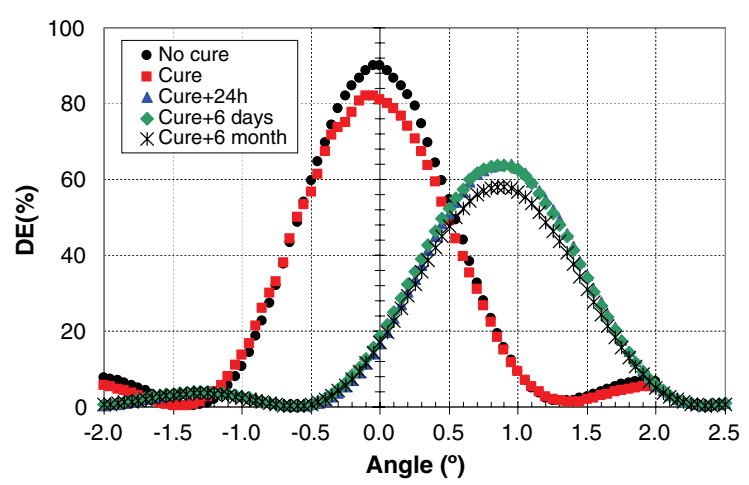

Fig. 4. DE versus angle of a transmission grating with a spatial frequency of 954 lines $/ \mathrm{mm}$ recorded in a photopolymer without ACPA.

Figure 4 shows the DE of a transmission grating stored in the photopolymer with composition 1 in Table 1, which does not contain ACPA. This grating was recorded with an exposure of $150 \mathrm{~mJ} / \mathrm{cm}^{2}$, at which the maximum DE was obtained. As shown, the DE just after storing the grating was $90 \%$ (black circles) and after curing it decreased slightly to $84 \%$ (red squares). However, $24 \mathrm{~h}$ later, the grating was reconstructed and the DE was seen to decrease to $64 \%$ (blue triangles), that is, it diminished by $29 \%$ of the initial value. The $\mathrm{DE}$ was measured again after 6 days to check whether it continued to decrease over time. As can be seen in Fig. 4 (green diamonds), the DE did not diminish further. The curing process contributed to fixing the grating, thereby preventing the stored information from being lost with time. In addition, 6 months after curing, the grating was exposed to the green laser beam and it was then reconstructed again. After this period of time, the DE diminished until a $58 \%$ (black asterisks).

With respect to shrinkage, the gratings reconstructed before and just after curing did not exhibit any shrinkage, since the two corresponding curves in Fig. 4 are centered on the zero of the abscissas axis, which corresponds to the Bragg angle. However, the gratings reconstructed $24 \mathrm{~h}, 6$ days, and 6 months after curing did exhibit shrinkage because the

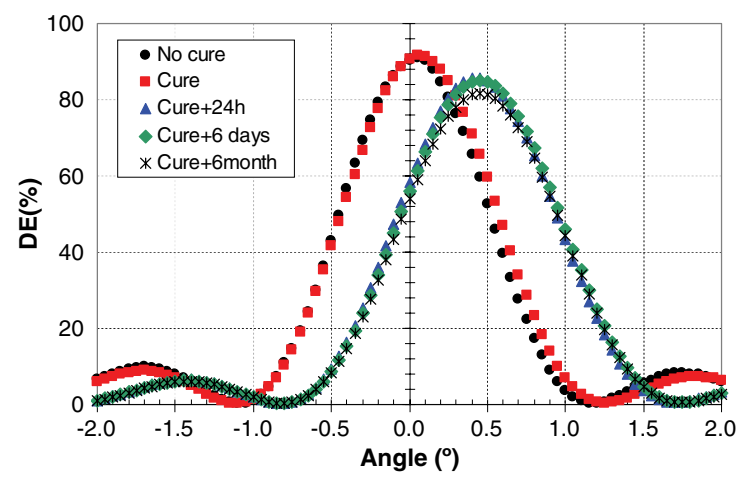

Fig. 5. DE versus angle of a transmission grating with a spatial frequency of 954 lines $/ \mathrm{mm}$ recorded in a photopolymer with ACPA. corresponding curves are displaced $0.9^{\circ}$ with respect to zero. This displacement, according to Eq. (ㅁ), corresponds to a $S_{\text {opt }}=5 \%$.

Figure $\underline{5}$ shows the DE versus reconstruction angle of the grating stored with a spatial frequency of 954 lines/mm but with composition 2 in Table 1 , which contains ACPA. This grating was recorded with an exposure of $150 \mathrm{~mJ} / \mathrm{cm}^{2}$, at which the maximum DE was obtained. The DE just after storage was $92 \%$ (black circles) and it remained at $92 \%$ after the curing process (red squares). Twenty-four hours (blue triangles) and 6 days (green diamonds) after curing, the DE was remeasured. As shown, the DE was the same for the two curves (85\%). In this case, the $\mathrm{DE}$ decreased by $7.6 \%$ of the initial value. Six months after curing and after the green laser beam exposition, DE was $82 \%$ (black asterisks).

The shrinkage was also calculated using Eq. (6), and a $S_{\text {opt }}=2.2 \%$ was obtained for the grating reconstructed $24 \mathrm{~h}, 6$ days, and 6 months after curing.

The reason why the DE decreased $24 \mathrm{~h}$ after curing, despite the fact that the dye had been removed, is that curing heats the material where the grating is stored and, as the material has a hydrophilic matrix, causes the water to evaporate, thus modifying the material. The evolution of the grating over time just after applying the curing process takes place as follows: During the early minutes after curing, the DE decreases more quickly, and after $60 \mathrm{~min}$ the DE decreases more slowly up to the moment when it stabilizes $6 \mathrm{~h}$ later. The same happens with the shrinkage process: Initially it increases more quickly (the peak of the grating is moving toward shorter wavelengths) and after $90 \mathrm{~min}$ the shrinkage remains almost constant. Consequently, this decrease of the DE and the increase of the shrinkage do not occur suddenly, but instead they are gradual processes that become faster just after applying the curing and they slow down as the minutes pass until they are fully stabilized $6 \mathrm{~h}$ later. Inasmuch as the final $\mathrm{DE}$ of the grating cannot be determined until the material is stable, the DE was measured $24 \mathrm{~h}$ after curing, the moment at which it can be ensured that the material reached stability and thus the DE remained constant when measured 6 days later, and it only experienced a slight decrease 6 months later. This heating also causes a slight shrinkage that produces a rotation of the Bragg fringes because the diffraction peaks at $24 \mathrm{~h}, 6$ days, and 6 months are displaced $0.9^{\circ}$ with respect to the Bragg angle, which would be centered at 0 on the graph.

When comparing Figs. 4 and 5, we can see that the $\mathrm{DE}$ after storage was just slightly superior for the grating recorded in composition 2 , which contained ACPA. Furthermore, this DE did not decrease after curing (Fig. 5), whereas the DE of the grating stored in composition 1 without ACPA did (Fig. 4). In Fig. 4, $24 \mathrm{~h}$ and 6 days after curing, the DE decreased by $29 \%$ of its initial value, whereas in Fig. $\underline{5}$, the DE decreased only $7.6 \%$. And 6 months later, the DE was $6 \%$ smaller than the DE obtained 6 days after curing 
for composition 1 (Fig. 4), and only a $3 \%$ smaller than the one for composition 2 (Fig. 5).

Therefore, the DE decreased by a much smaller percentage for composition 2 with ACPA than for composition 1 without ACPA. Furthermore, comparing the shrinkage $24 \mathrm{~h}, 6$ days, and 6 months after the curing process, for composition 2 with ACPA $\left(S_{\text {opt }}=2.2 \%\right)$, it was half of that for composition 1 without ACPA $\left(S_{\text {opt }}=5 \%\right)$. Therefore, ACPA contributed to fixing the stored gratings preventing a greater loss of information (higher DE) and producing a lower slant of Bragg fringes (lower shrinkage).

\section{B. Transmission Gratings with a Spatial Frequency of 2663 lines/mm}

Second, gratings with a spatial frequency of 2663 lines/mm were stored in the two compositions in Table 1 in order to see more clearly whether ACPA improves the resolution of the grating in addition to its fixation. The effect of ACPA on the stored gratings with this spatial frequency is shown in Figs. 6 and 7 . Again, each figure shows the DE of the grating just after recording (black circles), just after curing (red squares), and 24 h, 6 days, and 6 months after curing (blue triangles, green diamonds, and black asterisks, respectively). The zero on the abscissas axis corresponds to the Bragg angle for a wavelength of $633 \mathrm{~nm}$, which for this spatial frequency is $57.4^{\circ}$.

Figure $\underline{6}$ shows the $\mathrm{DE}$ versus reconstruction angle for a transmission grating stored at an exposure of $125 \mathrm{~mJ} / \mathrm{cm}^{2}$ (at which the maximum DE is obtained) in a photopolymer material without ACPA (composition 1). Immediately after recording the grating, the $\mathrm{DE}$ was $47 \%$ (black circles). Then the grating was subjected to a curing process and the $\mathrm{DE}$ when measured again was found to be $35 \%$ (red squares). Twenty-four hours after the curing process, the grating had a $\mathrm{DE}$ of $25 \%$ (blue triangles); 6 days after curing, the DE was still 25\% (green diamonds); and 6 months after curing and after the green laser beam exposure, the DE was $20 \%$ (black asterisks). Six days after the curing process the DE decreased $47 \%$ with respect to the initial DE before curing, and 6 months later, the $\mathrm{DE}$ diminished a $5 \%$ with

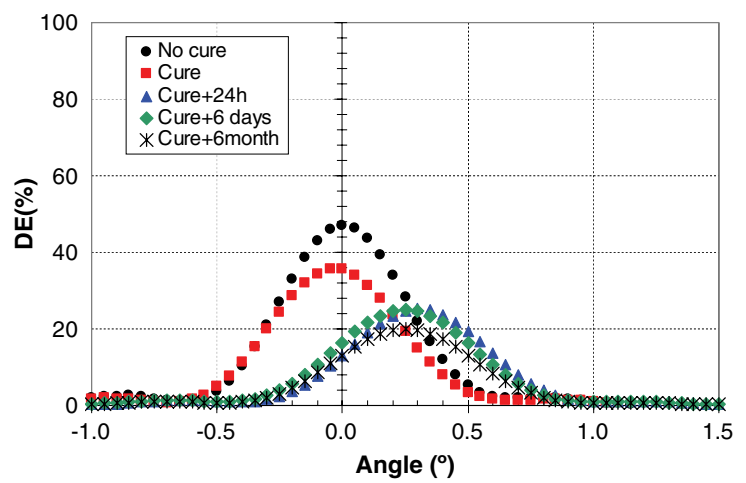

Fig. 6. DE versus angle of a transmission grating with a spatial frequency of 2663 lines/mm recorded in a photopolymer without ACPA.

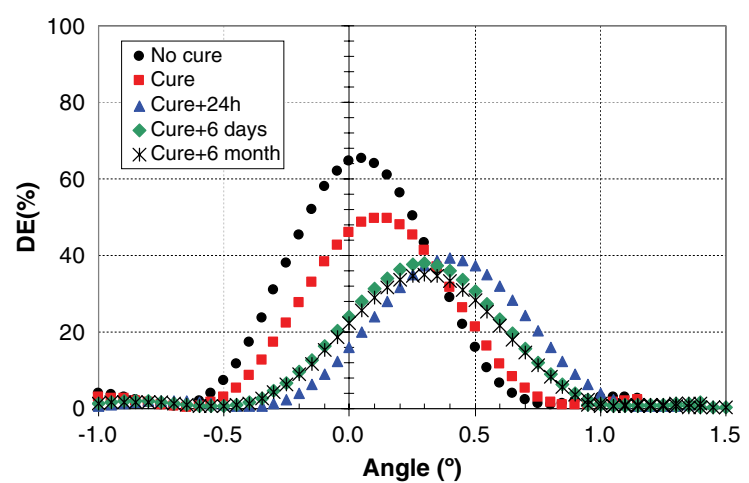

Fig. 7. DE versus angle of a transmission grating with a spatial frequency of 2663 lines $/ \mathrm{mm}$ recorded in a photopolymer with ACPA.

respect to the $\mathrm{DE}$ obtained 6 days after curing. There was no shrinkage of the grating before or just after curing, and $24 \mathrm{~h}$ and 6 days after curing, the maximum $\mathrm{DE}$ shifted $0.3^{\circ}$, which, according to Eq. (6), corresponds to a shrinkage of $S_{\text {opt }}=0.3 \%$.

Figure 7 shows the DE of a transmission grating stored in a photopolymer with ACPA (composition 2) at an exposure of $155 \mathrm{~mJ} / \mathrm{cm}^{2}$. In this case, the $\mathrm{DE}$ before curing was 65\% (black circles), after curing it was 50\% (red squares), after $24 \mathrm{~h}$ (blue triangles) and 6 days (green diamonds) it remained constant at $39 \%$. The DE decreased $40 \%$, with respect to the initial DE, due to the curing process. Six months after the curing the DE was 36\% (black asterisks). The shrinkage of the grating just after curing was $S_{\mathrm{opt}}=$ $0.17 \%$ and $24 \mathrm{~h}$ and 6 days after curing $S_{\mathrm{opt}}=0.4 \%$.

Comparing Figs. 6 and 7, it can be seen that the material without ACPA (composition 1) resolved this spatial frequency of 2663 lines/mm worse because the $\mathrm{DE}$ obtained was lower than in the case of the material with ACPA (composition 2). Therefore, incorporating ACPA in the material improved the resolution, resulting in a higher $\mathrm{DE}$, since it increased from $47 \%$ (without ACPA) to 65\% (with ACPA). The shrinkage in both compositions was small, between $0.3 \%$ and $0.4 \%$.

With respect to the stability of the gratings, the same process occurred with and without ACPA. Because of the curing process, the material was heated, and the DE decreased until the material cooled down completely. However once it was cold (at $24 \mathrm{~h}$ ) the material was stabilized and the DE remained constant. Six months later, the DE decreased $5 \%$ for composition 1 , and a $3 \%$ for composition 2 . Therefore, for both compositions the curing process stabilized, the behavior of the grating and maintained the DE constant once the material had cooled down. Nevertheless, the percentage decrease in DE due to the curing process and the lapse of 6 months was lower for the composition with ACPA (44\%) than for that without ACPA (57\%), so ACPA contributed to a better fixing of the grating after curing. The order of magnitude of shrinkage was the same for the two compositions. 


\section{Reflection Gratings with a Spatial Frequency of 4600 lines $/ \mathrm{mm}$}

Third, reflection gratings were stored with a spatial frequency of 4600 lines $/ \mathrm{mm}$, which is much higher than the previous two spatial frequencies. This was done to check whether ACPA also works for high spatial frequencies.

The experimental setup in Fig. 3 was used to store holographic reflection gratings with a spatial frequency of 4600 lines $/ \mathrm{mm}$. Gratings were recorded at different exposures and the $\mathrm{DE}$ was measured by a double-beam spectrophotometer. The spectrometer measures the transmittance of the photopolymer versus the wavelength. When the grating is not stored in the photopolymer, the transmittance curve has a shape-like that depicted in Fig. 1. However, an additional peak located at the Bragg wavelength appears after storing the grating, and this may be used to calculate its diffraction efficiency. Fixing the spatial period of the grating, which as mentioned in the previous section was $\Lambda_{\text {th }}=0.2174 \mu \mathrm{m}$, and knowing the refractive index of the material, which was $n_{633}=1.510$ for composition 1 and $n_{633}=$ 1.512 for composition 2, according to Eq. (2), the Bragg wavelength will depend on the reconstruction angle, $\theta$. In Fig. 1, the absorption peak of the material appears around $530 \mathrm{~nm}$. However, above $590 \mathrm{~nm}$ the photopolymer has a high transmittance and does not absorb anything. Therefore, if the grating was reconstructed with a suitable angle so that the diffraction peak had a Bragg wavelength of $590 \mathrm{~nm}$ or higher, it would not overlap with the absorption peak and would facilitate calculation of the $\mathrm{DE}$ of the grating. Therefore, the plate was placed perpendicular to the beam of the spectrophotometer $\left(\theta=0^{\circ}\right)$ and so the Bragg wavelength according to Eq. (2) was $656 \mathrm{~nm}$ for composition 1 and $655 \mathrm{~nm}$ for composition 2 . The measurements were not carried out in real-time, so once the grating was stored in the material, it was reconstructed with the spectrophotometer. The diffraction peak appears at these wavelengths.

After storing the gratings in the material at different exposures, their transmittance was measured with the spectrophotometer following the procedure described. The gratings were reconstructed in a spectral range (610-660 $\mathrm{nm}$ ) where absorption is negligible, so the conservation of energy principle implies that transmittance $T(\lambda)$ and reflectance $R(\lambda)$ are approximately complementary, i.e., $T(\lambda)+R(\lambda) \approx$ 1 and thus $R(\lambda) \approx 1-T(\lambda)$ ). Since the spectrophotometer gives the energy transmitted, analyzing just the transmittance $T(\lambda)$ will suffice. Once the transmittance is known, the DE is defined as the depth of this transmitted peak and can be calculated from Eq. (ㅇ) [미, $\underline{24}]$ :

$$
\mathrm{DE}=\frac{T_{p}-T_{p g}}{T_{p}},
$$

where $T_{p}$ is the transmittance of the photopolymer layer without the recorded grating and $T_{p g}$ the

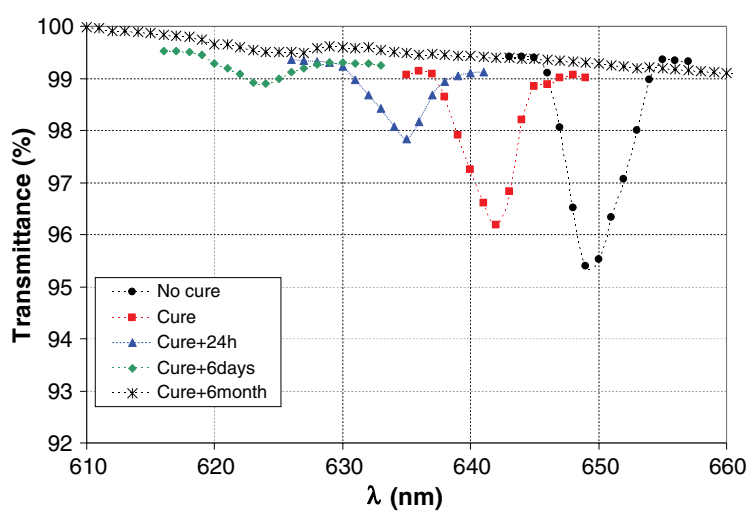

Fig. 8. Transmittance as a function of wavelength for a reflection grating with a spatial frequency of 4600 lines $/ \mathrm{mm}$ recorded in a photopolymer without ACPA.

transmittance of the photopolymer layer with the recorded grating.

Figures $\underline{8}$ and 9 show the transmittance of the gratings stored with the exposure that gave maximum DE for composition 1 without ACPA that was (Fig. 8) and composition 2 with ACPA (Fig. 9). The sample without ACPA was recorded with $360 \mathrm{~mJ} / \mathrm{cm}^{2}$ whereas the sample with ACPA was recorded with $340 \mathrm{~mJ} / \mathrm{cm}^{2}$. Both figures show the transmittance of the gratings immediately after recording (black circles), just after curing (red squares), $24 \mathrm{~h}$ after curing (blue triangles), 6 days after curing (green diamonds), and 6 months after curing and after green laser beam exposure (black asterisks). With the transmittance values in the two figures, the $\mathrm{DE}$ is calculated from Eq. (8). And with the wavelength at which the diffraction peaks are located $\left(\lambda_{\exp }\right)$ and the Bragg wavelength calculated above in Section 2.C $\left(\lambda_{\text {th }}=656 \mathrm{~nm}\right.$ for composition 1 and $\lambda_{\text {th }}=655 \mathrm{~nm}$ for composition 2 ), the shrinkage can be calculated from Eq. (7).

Using the transmittance results in Fig. 8 , the DE was $4 \%$ before curing, 3\% after curing, $1.5 \% 24 \mathrm{~h}$ after curing, $0.5 \% 6$ days after curing, and null 6 months after curing. In this case, 6 days after curing the DE decreased by $87.5 \%$ with respect to the initial value obtained just after storing the grating and the $\mathrm{DE}$ is equal to zero 6 months after curing.

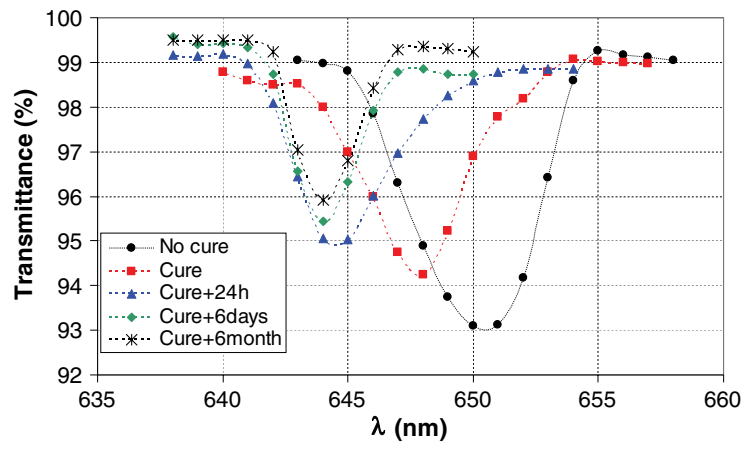

Fig. 9. Transmittance as a function of wavelength for a reflection grating with a spatial frequency of 4613 lines $/ \mathrm{mm}$ recorded in a plate with ACPA. 
In addition, from the position of the peaks and the $\lambda_{\text {th }}=656 \mathrm{~nm}$ corresponding to composition 1 , the shrinkage was $S_{\text {opt }}=1 \%$ before curing, $S_{\text {opt }}=2.1 \%$ after curing, $S_{\text {opt }}=3.2 \% 24 \mathrm{~h}$ after curing, and $S_{\text {opt }}=5 \% 6$ days after curing.

From the transmittance data in Fig. 9, the DE was $6.2 \%$ just after storing the grating, $4.4 \%$ just after the curing process, $4 \% 24 \mathrm{~h}$ after curing, $3.8 \% 6$ days after curing, and 3.5\% 6 months after curing. In this case, during the 6 months after curing the $\mathrm{DE}$ decreased by $43.5 \%$ with respect to the value initially obtained after recording the grating. The shrinkage was $S_{\text {opt }}=0.6 \%$ immediately after recording, $S_{\text {opt }}=$ $1 \%$ just after curing the grating, $S_{\text {opt }}=1.5 \% 24 \mathrm{~h}$ after curing, and $S_{\text {opt }}=1.7 \% 6$ days after curing.

Comparing the data in Figs. 8 and 9 we can see that for the grating stored in composition 1 without ACPA (Fig. 8), the DE decreased as time went by from $4 \%$ (just after recording the grating) to almost zero ( 6 days after the recording), despite the fact that a curing process was used to fix the grating. However, for a grating recorded in composition 2 with ACPA (Fig. 9), the DE of the grating after recording was $6.2 \%$, which is higher than that obtained in Fig. 8 . Moreover, $24 \mathrm{~h}, 6$ days, and 6 months after the curing process, the $\mathrm{DE}$ of the grating was not close to zero as in Fig. 8, but remained constant between $4 \%$ and $3 \%$. Therefore, ACPA, in combination with the curing process, contributed to the fixing of the grating.

In addition, Fig. $\underline{8}$ (without ACPA) shows that as time passed, the shrinkage increased from $1 \%$ just after recording the grating, to $5 \% 6$ days after curing. However, from the results in Fig. 9 (with ACPA), the shrinkage increased only slightly from $0.6 \%$ (just after recording the grating) to $1.7 \%$ (6 days after curing), and this value was maintained over time. Therefore, ACPA reduced the shrinkage.

\section{Conclusions}

This study has demonstrated the validity of using chain transfer agents, specifically ACPA, to increase the spatial resolution of photopolymers, reduce shrinkage, and improve the process of fixing the information after the surplus dye in the material has been removed by a curing process.

ACPA improved the spatial resolution of the material since transmission gratings stored with a spatial frequency of 2663 lines/mm in composition 2 with ACPA had a higher DE (65\%) than those stored in composition 1 without ACPA ( $\mathrm{DE}=47 \%)$. Moreover, for reflection gratings with a spatial frequency of 4600 lines $/ \mathrm{mm}$, the $\mathrm{DE}$ increased from $4 \%$ (without ACPA) to $6.2 \%$ (with ACPA). In the case of 954 lines $/ \mathrm{mm}$, a $\mathrm{DE}=90 \%$ was reached with both compositions. From these results it may be concluded that the photopolymer without ACPA has greater difficulty in resolving gratings with high frequencies (greater than 2500 lines $/ \mathrm{mm}$ ), which results in a low DE. Incorporating ACPA in the composition of the photopolymer increases the resolution of the material yielding a higher DE. Therefore, ACPA improves the resolution of the material.

For the spatial frequencies studied, there was less shrinkage in composition 2 with ACPA than in composition 1 without ACPA. For 954 lines $/ \mathrm{mm}$, the shrinkage decreased from $S_{\text {opt }}=5 \%$ for composition 1 without ACPA to $S_{\text {opt }}=2.2 \%$ for composition 2 with ACPA. For 2663 lines $/ \mathrm{mm}$, the shrinkage was approximately constant around $0.3 \%$. And for 4600 lines $/ \mathrm{mm}$, the shrinkage decreased from $S_{\text {opt }}=$ $5 \%$ (without ACPA) to $S_{\text {opt }}=1.7 \%$ (with ACPA).

With respect to the stability of the gratings, ACPA improved the fixation of the gratings for the three spatial frequencies studied, since in the three cases in the composition with ACPA the decrease in DE after the curing process was smaller than that in the composition without ACPA.

This work was supported by the "Generalitat Valenciana" (Spain) under project PROMETEO/2011/ 021, ISIC/2012/013, and GV/2012/099 and by "Ministerio de Ciencia e Innovación" (Spain) under projects FIS2011-29803-C02-01 and FIS2011-29803-C02-02.

\section{References}

1. E. Fernández, M. Ortuño, S. Gallego, C. García, A. Beléndez, and I. Pascual, "Comparison of peristrophic multiplexing and a combination of angular and peristrophic holographic multiplexing in a thick PVA/acrylamide photopolymer for data storage," Appl. Opt. 46, 5368-5373 (2007).

2. E. Fernández, M. Ortuño, S. Gallego, A. Márquez, C. García A. Beléndez, and I. Pascual, "Multiplexed holographic data page storage on a PVA/acrylamide photopolymer memory," Appl. Opt. 47, 4448-4456 (2008).

3. L. Dhar, K. Curtis, and T. Facke, "Holo graphic data storage: coming of age," Nat. Photonics 2, 403-405 (2008).

4. D. Graham-Rowe, "The drive for holography," Nat. Photonics 1, 197-200 (2007).

5. K. Curtis, L. Dhar, A. Hill, W. Wilson, and M. Ayres, Holographic Data Storage: From Theory to Practical Systems (Wiley, 2010).

6. V. A. Barachevskii, "Photopolymerizable recording media for three-dimensional holographic optical memory," High Energy Chem. 40, 131-141 (2006).

7. R. C. Fontanilla-Urdaneta, M. P. Hernandez-Garay, A. Olivares-Perez, G. Paez-Trujillo, and I. Fuentes-Tapia, "Diffraction efficiency study of holographic gratings in dichromated poly(vinyl alcohol) $\mathrm{NiCl}_{2}$ center $\operatorname{dot} 6 \mathrm{H}(2) \mathrm{O}$ doped-art . no. 691206," Prac. Hologr. XXII: Mater. Appl. 6912, 91206 (2008).

8. S. H. Lin, P. L. Chen, Y. N. Hsiao, and W. T. Whang, "Fabrication and characterization of poly(methyl methacrylate) photopolymer doped with 9,10-phenanthrenequinone (PQ) based derivatives for volume holographic data storage," Opt. Commun. 281, 559-566 (2008).

9. I. Naydenova, S. Martin, R. Jallapuram, R. Howard, and V. Toal, "Investigations of the diffusion processes in self-processing acrylamide-based photopolymer system," Appl. Opt. 43, 2900-2905 (2004).

10. S. Martin, I. Naydenova, V. Toal, R. Jallapuram, and R. Howard, "Two way diffusion model for the recording mechanism in a self developing dry acrylamide photopolymer," Proc. SPIE 6252, 37-44 (2006).

11. J. Guo, M. R. Gleeson, S. Liu, and J. Sheridan, "Non-local spatial frequency response of photopolymer materials containing chain transfer agents: II. Experimental results," J. Opt. 13, 095602 (2011).

12. R. Fuentes, E. Fernandez, C. Garcia, A. Belendez, and I. Pascual, "Study of influence of ACPA in holographic reflection 
gratings recorded in PVA/AA based photopolymer," Proc. SPIE 7717, 77170Q (2010).

13. I. Naydenova, R. Jallapuram, V. Toal, and S. Martin, "A visual indication of environmental humidity using a color changing hologram recorded in a self-developing photopolymer," Appl. Phys. Lett. 92, 031109 (2008).

14. I. Naydenova, R. Jallapuram, V. Toal, and S. Martin, "Characterisation of the humidity and temperature responses of a reflection hologram recorded in acrylamide-based photopolymer," Sens. Actuators B 139, 35-38 (2009).

15. M. Ortuno, E. Fernandez, R. Fuentes, S. Gallego, I. Pascual, and A. Belendez, "Improving the performance of PVA/AA photopolymers for holographic recording," Opt. Mater. 35, 668-673 (2013).

16. E. Fernandez, A. Marquez, S. Gallego, R. Fuentes, C. García, and I. Pascual, "Hybrid ternary modulation applied to multiplexing holograms in photopolymers for data page storage," J. Lightwave Technol. 28, 776-783 (2010).

17. M. Ortuño, A. Marquez, E. Fernández, S. Gallego, A. Belendez, and I. Pascual, "Hologram multiplexing in acrylamide hydrophilic photopolymers," Opt. Commun. 281, 1354-1357 (2008).
18. M. Weiser, F. Bruder, T. Facke, D. Honel, D. Jurbergs, and T. Rolle, "Self-processing, diffusion-based photopolymers for holographic applications," Macromol. Symp. 296, 133-137 (2010).

19. S. Gallego, C. Neipp, M. Ortuño, A. Beléndez, and I. Pascual, "Stabilization of volume gratings recorded in polyvinyl alcohol-acrylamide photopolymers with diffraction efficiencies higher than 90\%," J. Mod. Opt. 51, 491-503 (2004).

20. J. A. Jenney, "Holographic recording with photopolymers," J. Opt. Soc. Am. 60, 1155-1161 (1970).

21. R. J. Collier, C. B. Burckhardt, and L. H. Lin, Optical Holography (Academic, 1971).

22. J. T. Gallo and C. M. Verber, "Model for the effects of material shrinkage on volume holograms," Appl. Opt. 33, 6797-6804 (1994).

23. L. Criante, K. Beev, D. E. Lucchetta, and F. Simoni, "Spectral analysis of shrinkage in holographic materials suitable for optical storage applications," Proc. SPIE 6252, 62520G (2006).

24. R. Fuentes, E. Fernandez, C. Garcia, A. Belendez, and I. Pascual, "Study of reflection gratings recorded in polyvinyl alcohol/acrylamide-based photopolymer," Appl. Opt. 48 6553-6557 (2009). 